\title{
Comet Scoring Engine
}

National Cancer Institute

\section{Source}

National Cancer Institute. Comet Scoring Engine. NCI Thesaurus. Code C68827.

A private scoring eng ine developed by the Fred Hutchinson Cancer Research Institute that can match tandem mass spectra with peptide sequences. 